\title{
COMMUNICATIONS
}

\section{The solid-liquid interfacial free energy of close-packed metals: Hard-spheres and the Turnbull coefficient}

\author{
Brian B. Laird \\ Department of Chemistry, University of Kansas, Lawrence, Kansas 66045
}

(Received 2 May 2001; accepted 20 June 2001)

\begin{abstract}
Largely due to its role in nucleation and crystal-growth, the free energy of the crystal-melt interfacial free energy is an object of considerable interest across a number of scientific disciplines, especially in the materials-, colloid-, and atmospheric sciences. Over 50 years ago, Turnbull observed that the interfacial free energies (scaled by the mean interfacial area per particle) of a variety of metallic elements exhibit a linear correlation with the enthalpy of fusion. This correlation provides an important empirical "rule-of-thumb" for estimating interfacial free energies, but lacks a compelling physical explanation. In this work we show that the interfacial free energies for close-packed metals are linearly correlated with the melting temperature and are therefore primarily entropic in origin. We also show that the slope of this linear relationship can be determined with quantitative accuracy using a hard-sphere model, and that the correlation with the enthalpy of fusion reported by Turnbull follows as a consequence of the fact that the entropy of fusion for close-packed metals is relatively constant. (C) 2001 American Institute of Physics. [DOI: 10.1063/1.1391481]
\end{abstract}

The crystal-melt interfacial free energy, $\gamma$, defined as the reversible work required to form a unit area of interface between a crystal and its coexisting fluid, plays a central role in determining the kinetics of crystal nucleation and growth. ${ }^{1,2}$ Unfortunately, direct experimental determinations of this quantity are difficult and exist for only a handful of materials. For most materials, knowledge of the interfacial free energy is obtained indirectly from measurements of crystal nucleation rates from undercooled fluids from which $\gamma$ is determined within the approximations of classical nucleation theory.

In a seminal 1950 paper, ${ }^{3}$ Turnbull reported values of the crystal-melt interfacial free energy, $\gamma$, for a variety of materials, mostly metallic elements, which were obtained indirectly from nucleation rate experiments. For systems in which directly determined values exist with which to compare the values so obtained typically are accurate within about 10-20\%. (For example, the interfacial free energy for bismuth was determined ${ }^{4}$ using grain boundary angles to be $61.3 \times 10^{-3} \mathrm{~J} \mathrm{~m}^{-2}$, as compared to the value of 54.4 $\times 10^{-3} \mathrm{~J} \mathrm{~m}^{-2}$ obtained from nucleation rate data.) In order to compare the results for various systems, Turnbull defined a "gram-atomic" (or molar) interfacial free energy as the free energy of an interface (one atom thick) containing Avagadro's number, $N_{A}$, of atoms (or molecules):

$$
\hat{\gamma}=\gamma \rho^{-2 / 3} N_{A}
$$

The data for $\hat{\gamma}$ was found to exhibit a strong correlation with the latent heat of fusion. Empirically, Turnbull found

$$
\hat{\gamma}=C_{T} \Delta_{f u s} H,
$$

where the Turnbull coefficient, $C_{T}$, was found to be approximately 0.45 for metals (especially close-packed metals) and 0.32 for many nonmetals.

There have been attempts to explain this empirical result through the analysis of simple models for the structure of the interface, ${ }^{5-7}$ but the results are quite sensitive to the nature of the assumed model. Inherent in these early models are two basic assumptions: (1) the solid-liquid free energy is primarily entropic in origin and (2) the surface free energy is due to the increase in entropy associated with the enhanced structure of the liquid at the interface (i.e., the crystal is unchanged up to the interface). The first assumption is reasonable given the dominant role that packing considerations play in determining the structure and thermodynamics of simple liquids ${ }^{8}$ and implies that a hard-sphere model should be adequate to describe the interfacial system. However, the assumption (2) is at variance with molecular-dynamics simulations of crystal-melt interfaces of simple systems ${ }^{9,10}$ that show significant structural relaxation (evidenced by an increase in the mean-squared displacement from the lattice sites) occurs in the crystal as the interface is approached. Therefore, any theory of the crystal-melt interfacial free energy must include a realistic description of both the solid and fluid in the interfacial region.

Recently, we have determined via molecular-dynamics computer simulation, the structure ${ }^{10}$ and solid-liquid interfacial free energy ${ }^{11}$ for a system of hard-spheres. For this system, which freezes into a face-centered-cubic (fcc) crystal structure, $\gamma$ was determined to be slightly anisotropic with an orientationally averaged value of

$$
\gamma_{h s}=0.61 k T_{m} / \sigma^{2},
$$

where $k$ is Boltzmann's constant, $T_{m}$ is the melting tempera- 


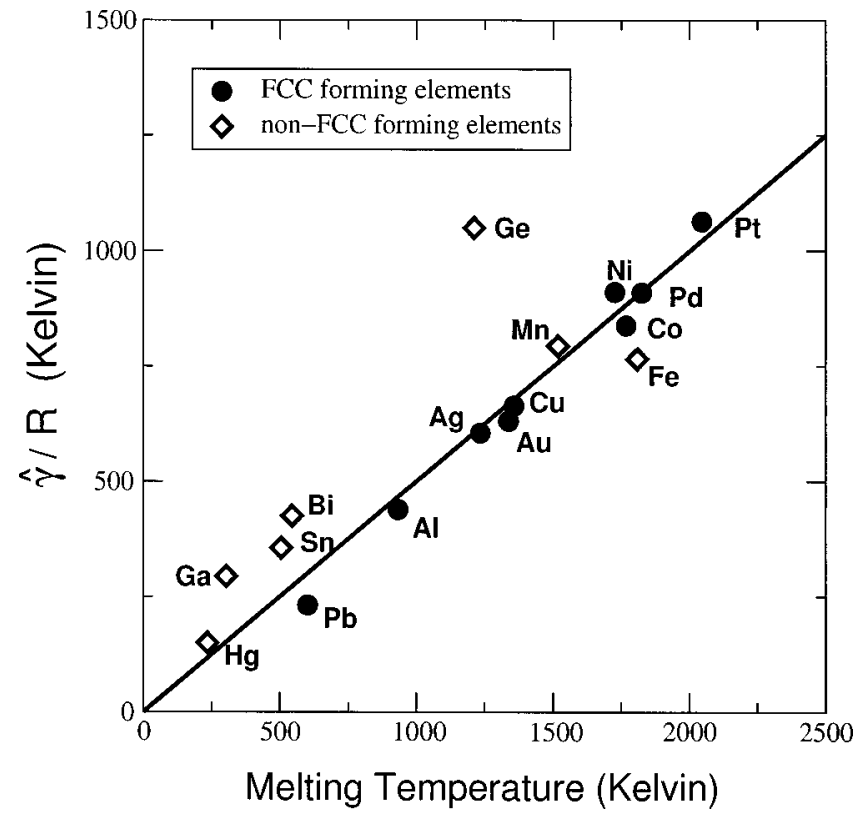

FIG. 1. Gram-atomic interfacial free energy (scaled by the gas constant to give units of Kelvin) for a variety of elemental systems. fcc-crystal-forming systems are shown as filled circles and values for non-close-packed crystal formers are shown as open diamonds. The dashed line is a line of slope 0.50 representing the best linear fit passing through the origin to the data for the fcc crystal formers.

ture, and $\sigma$ is the hard-sphere diameter. (The scaling with $k T_{m}$ is a consequence of the fact that the phase behavior in any hard-core system is purely entropic.) This value is consistent with a value of $0.55 \pm 0.02 k T_{m} / \sigma^{2}$ obtained from an analysis $^{12}$ (using nucleation theory) of the experimental crystallization kinetics of silica spheres, a system well described by a hard-sphere model. Note that $\gamma_{h s}$ is considerably lower than that obtained for a fluid at a structureless hard wall which was recently calculated ${ }^{14}$ at the melting density to be $1.99 \pm 0.18 k T_{m} / \sigma^{2}$, indicating that the entropy increase due to the relaxation of the crystal structure near the interface plays an important role in determining the interfacial thermodynamics.

For the hard-sphere system, the density of the solid at freezing is approximately $\rho \sigma^{3}=1.04$, independent of temperature, ${ }^{13}$ therefore Turnbull's gram-atomic interfacial free energy can be written

$$
\hat{\gamma}_{h s} / R=0.59(0.54) T_{m},
$$

where $R$ is the gas constant and the value in parentheses is that obtained using the value of $\gamma_{h s}$ determined from nucleation experiments. ${ }^{12}$ Equation (4) suggests that if the interfacial free energy of fcc-forming materials is well described by a hard-sphere model, then one should see a linear correlation between $\hat{\gamma} / R$ and the melting temperature with a slope in the range 0.5 to 0.6 .

To test this hypothesis we plot in Fig. $1 \hat{\gamma} / R$ as a function of melting temperature for a variety of elemental materials using Turnbull's original data. Although all of the data exhibits a correlation with $T_{m}$, the correlation for the fcc- crystal forming metals is linear with a slope of 0.50 , whereas that for non-fcc forming materials exhibits significant scatter. This value of the slope is about $20 \%$ below that predicted by the direct hard-sphere results but less than $10 \%$ below that based on the nucleation rate result, which is probably a more relevant comparison since Turnbull's data was also so obtained. That the attractive forces contribute only about $10 \%$ to the interfacial free energy is consistent with results from simulation $^{9,11}$ and density-functional theory ${ }^{15}$ for the Lennard-Jones system, a prototypical fcc-forming model potential. It should be noted that Turnbull also reported ${ }^{3}$ a correlation of $\hat{\gamma}$ with $T_{m}$, but, due to the scatter in the overall data, it was rejected as the basis for an empirical rule in favor of the correlation with the enthalpy of fusion. However, he did observe that the correlation with $T_{m}$ was sensitive to the "complexity" of the crystal structure. This is evident in the data shown in Fig. 1, which shows a strong linear correlation with $T_{m}$, for fcc-forming metals, whereas that for the nonclose-packed materials is much weaker. The hard-sphere interaction does not have the long-range forces necessary to mechanically stabilize a non-close packed crystal structure. As a consequence, the interfacial thermodynamics of systems that freeze into open crystal lattices will not be well described by a purely entropic model and a simple linear scaling with the melting temperature is not expected.

For fcc- (and probably hcp-) forming materials, it is relatively straightforward now to understand the empirical correlation of the interfacial free energy with the heat of fusion. For the close-packed systems studied by Turnbull, the entropy of fusion, $\Delta_{f u s} S$, is very nearly constant with an average value very close to that for the hard-sphere system, where $\Delta_{f u s} S=9.7 \mathrm{~J} /($ mole $\mathrm{K})$. At the melting point, equilibrium requires that $\Delta_{f u s} H=T_{m} \Delta_{f u s} S$, so the enthalpy of fusion should scale nearly linearly with the melting temperature for these systems. So if $\Delta_{f u s} H$ scales approximately linear with $T_{m}$ and $\hat{\gamma}_{h s}$ is proportional to $T_{m}$, it then follows that $\hat{\gamma}_{h s}$ will exhibit strong linear correlation with $\Delta_{f u s} H$, and thus Turnbull's rule obtains.

The author gratefully acknowledges Dr. Ruslan Davidchack for helpful conversations and the National Science Foundation for generosity under Grant No. CHE-9970903.

${ }^{1}$ D. P. Woodruff, The Solid-Liquid Interface (Cambridge University Press, Cambridge, 1973)

${ }^{2}$ J. M. Howe, Interfaces in Materials (Wiley, New York, 1997).

${ }^{3}$ D. Turnbull, J. Appl. Phys. 21, 1022 (1950).

${ }^{4}$ M. E. Glicksman and C. Vold, Acta Metall. 17, 1 (1969).

${ }^{5}$ A. Skapski, Acta Metall. 4, 583 (1956).

${ }^{6}$ F. Spaepen, Acta Metall. 23, 729 (1975).

${ }^{7}$ F. Spaepen, Scr. Metall. 10, 257 (1976).

${ }^{8}$ J.-P. Hansen and I. R. McDonald, Theory of Simple Liquids (Cambridge University Press, Cambridge, 1986).

${ }^{9}$ J. Q. Broughton and G. H. Gilmer, J. Chem. Phys. 84, 5759 (1986).

${ }^{10}$ R. L. Davidchack and B. B. Laird, J. Chem. Phys. 108, 9452 (1998).

${ }^{11}$ R. L. Davidchack and B. B. Laird, Phys. Rev. Lett. 85, 4751 (2000).

${ }^{12}$ D. W. Marr and A. P. Gast, Langmuir 10, 1348 (1994).

${ }^{13}$ W. G. Hoover and F. H. Ree, J. Chem. Phys. 49, 3609 (1968).

${ }^{14}$ M. Heni and H. Löwen, Phys. Rev. E 60, 7057 (1999).

${ }^{15}$ W. A. Curtin, Phys. Rev. B 39, 6775 (1989). 